\title{
In Situ Observation of Pt Icosahedral Nanoparticles Transformation into FCC Single Crystal
}

\author{
Wenpei Gao ${ }^{1,2}$, Jianbo Wu ${ }^{1,2,3}$, Hyuk Park ${ }^{1,2}$, J. Mabon ${ }^{2}$, W.L. Wilson², Hong Yang ${ }^{3}$, Jian-Min Zuo ${ }^{1,2}$ \\ 1. Dept. of Materials Science and Engineering, University of Illinois, Urbana-Champaign, IL 61801 \\ 2. Fredrick Seitz Materials Research Laboratory, University of Illinois, Urbana-Champaign, IL 61801 \\ 3. Dept. of Chemical \& Biomolecular Engineering, University of Illinois, Urbana-Champaign, IL 61801
}

Significant progress has been made in spatial resolution using environmental transmission electron microscopes (ETEM), which now enables atomic resolution visualization of structural transformation under variable temperature and gas environments close to materials' real operational conditions (for a review, see ref [1]). Here we report the observation of transformation of Pt icosahedral nanoparticles into FCC single crystals upon heating in oxygen, at $0.11 \mathrm{~nm}$ spatial resolution and $2.5 \mathrm{~ms}$ time resolution.

Icosahedral nanoparticles have attracted special interest in nanoscience and technology because of their unusual atomic packing and noncrystallographic symmetry. They are predicted to be unstable compared to the bulk FCC structure at large sizes. Recently, uniformly large icosahedral nanoparticles of 20 to 36 $\mathrm{nm}$ have been successfully synthesized through careful control of growth kinetics and demonstrate outstanding chemical activities for fuel cell catalysis [2,3]. However, experimental evidences suggest that those icosahedral structure is unstable when exposed to oxygen at elevated temperatures. To investigate this and to address the critical question of transformation between the metastable MTPs and stable FCC structure, we carried out an in-situ study using an environmental TEM with fast electron imaging capability.

Experiments were carried out on Hitachi H-9500 ETEM with a $\mathrm{LaB}_{6}$ emitter, operated at $300 \mathrm{kV}$. The instrument provides direct gas injection into sample chamber as well as gas injection through a specially designed sample holder. The samples are heated using a W filament by passing current. The direct electron detection camera (model K2-IS) made by Gatan (Pleasanton, CA) is used for video recording, providing improved time resolution of $2.5 \mathrm{~ms}$ (milliseconds) for full frame or $0.625 \mathrm{~ms}$ for $1 / 4$ frames.

Figure 1 shows a cluster of Pt icosahedral nanoparticles before and after phase transformation, upon heating in oxygen at elevated temperatures. In Figure 1a, recorded at the beginning of the experiment, all nanoparticles were faceted showing their characteristic hexagonal shapes. The icosahedral structure is evidenced by the diffraction pattern in Figure 1e obtained from the particle in Figure 1d. After the transformation, the particles showed rounded shapes as well as evidences of coalescence. The diffraction pattern recorded from the particles indicates that they are FCC crystal of the same orientation. Unlike these previous reports showing that small MTPs do not transform into the same structure, due to the small energy difference among different configurations, we observed that all the IC NPs transform into a single FCC crystal. Details of the experiment and results will be provided to bring better understanding of the mechanisms.

\section{References:}

[1] J.R. Jinschek, Chemical Communications, 50(21) (2014), p. 2696-2706. 
[2] W. Zhou, J. Wu, and H. Yang, Nano Letters, 13(6) (2013), p. 2870-2874.

[3] J. Wu, et al., Nano Letters, 15(4) (2015), p. 2711-2715.

[4] Wenpei Gao was supported by NSF DMR-1410596. The development of ETEM was supported by the NSF MRI Grant NSF DMR 12-29454 and University of Illinois.
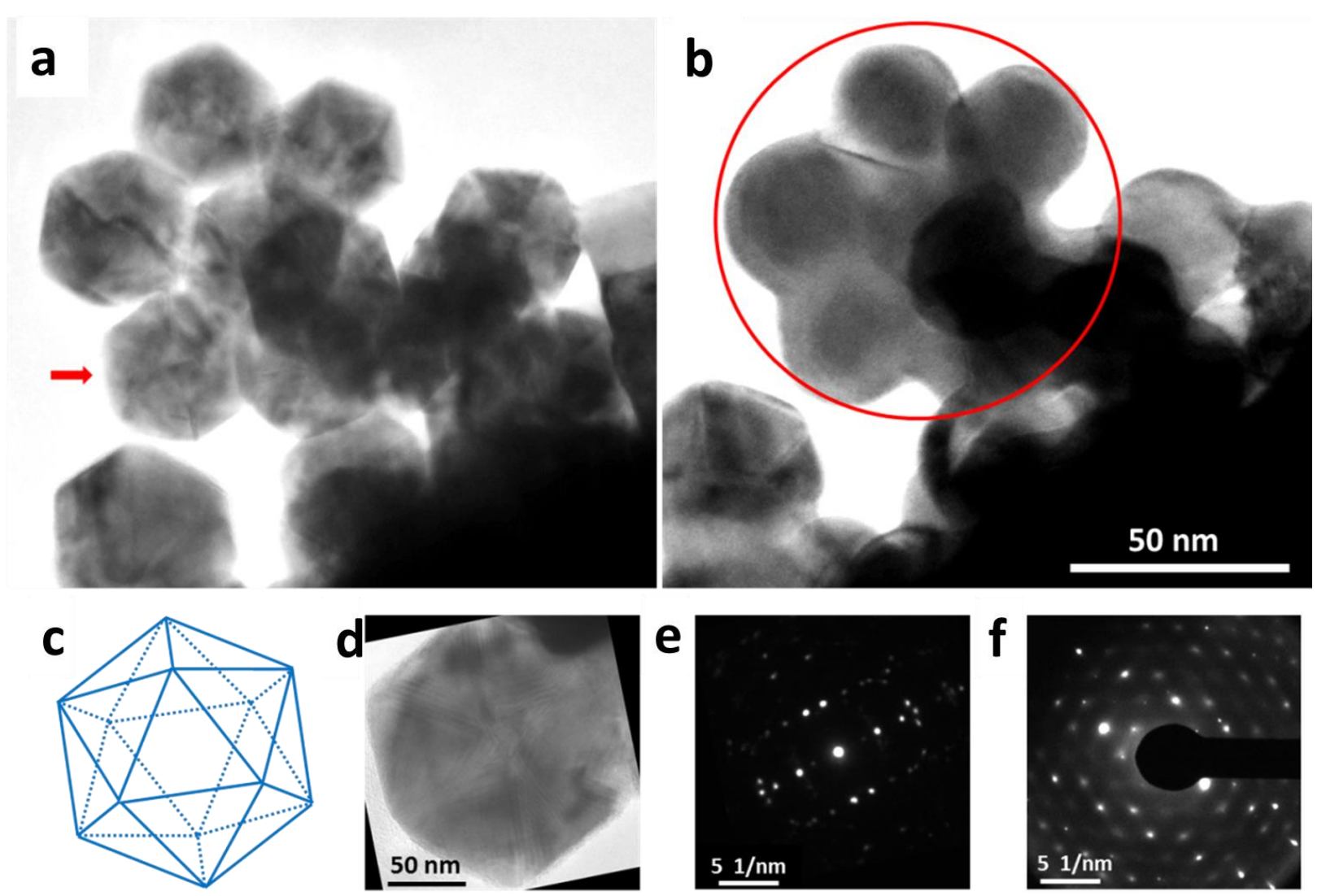

Figure 1. Transformation of a group of Pt IC NPs to FCC crystal as demonstrated by electron images recorded (a) before and (b) after the transformation. The IC NPs were oriented approximately as illustrated in (c). The IC structure is evidenced by electron diffraction (e) recorded from (d), the particle selected in (a) by the red arrow. (f) is an electron diffraction pattern recorded from the transformed NPs using a probe indicated by the circle in $b$ ). 